\title{
Fertilidade do solo em pastagens cultivadas sob diferentes manejos, no noroeste do Rio de Janeiro ${ }^{1}$
}

\author{
Leonardo M. M. Pimenta ${ }^{2}$, Everaldo Zonta ${ }^{3}$, Felipe C. Brasil ${ }^{4}$, Lucia H. C. dos Anjos ${ }^{3}$, Marcos G. Pereira ${ }^{3} \&$ Juliano B. Stafanato ${ }^{3}$
}

\section{RESUMO}

Com o objetivo de avaliar os efeitos de diferentes espécies forrageiras e práticas de manejo sobre a variação da fertilidade do solo conduziu-se um experimento no município de Itaperuna, na Região Noroeste do Estado do Rio de Janeiro. Três piquetes de 2 ha foram divididos em um Argissolo Vermelho-Amarelo em relevo forte ondulado, cultivado inicialmente com Brachiaria brizantha. Além da manutenção desta espécie foram implantadas as espécies Tifton 85 (Cynodon spp.) e Suázi (Digitaria swazilandensis) perfazendo três tratamentos que receberam adubação anual a partir da instalação do experimento (2001). Realizaram-se nove coletas de solo em três terços de cada piquete (médio, inferior e superior) entre os meses de abril de 2002 e maio de 2006 e se coletaram amostras de $1 \mathrm{dm}^{3}$ de solo, em seções de 0,1 até $0,7 \mathrm{~m}$, com duas repetições por trincheira. Foram analisados e interpretados, em cada data de coleta, todos os parâmetros de fertilidade do solo. Os dados demonstraram que, após a introdução das novas espécies, do manejo rotacionado e das adubações de manutenção, os parâmetros de fertilidade mantiveram valores muito próximos sem haver um decréscimo ao longo do tempo na área experimental.

Palavras-chave: manejo rotacionado, recuperação de pastagens degradadas

\section{Soil fertility of cultivated pastures under different managements in the northwest Rio de Janeiro}

\begin{abstract}
Aiming to evaluate the effects of different grass species and management practices on soil fertility, an experiment was conducted in the municipality of Itaperuna, northwest part of Rio de Janeiro State. Three plots of 2 ha were divided in a Red Yellow Ultisol with a strong ondulating relief originally cultivated with Brachiaria brizantha. Besides the maintenance of this specie, the Tifton 85 (Cynodon spp.) and Suázi (Digitaria swazilandensis) were introduced, performing three treatments which received annual fertilization from the beginning of the experiment (2001). N ine soil samplings were done in three thirds of each plot (medium, inferior and superior) between A pril 2002 and May 2006. Soil samples of $1 \mathrm{dm}^{3}$ were taken from $0.1 \mathrm{~m}$ to $0.7 \mathrm{~m}$ depths, with 2 repetitions per trench. Soil fertility attributes were analyzed and interpreted in each sampling time. The data show that, after the introduction of the new species, the rotationed management and the maintenance fertilization, the soil fertility attributes kept very close values without decreasing along time in the experimental area.
\end{abstract}

Key words: rotationed management, recovery of degraded pastures

\footnotetext{
1 Parte da Dissertação de Mestrado do primeiro autor, apresentada a Universidade Federal Rural do Rio de Janeiro

${ }^{2}$ Zootecnista, Mestre em Agronomia, Rua Maestro Francisco Braga 295/102 Copacabana, CEP 22041-070, Rio de Janeiro, RJ. Fone: (21) 2236-4976. E-mail leomorucci@hotmail.com

${ }^{3}$ DS/U FRRJ, BR-465 km 7, CEP 23890 000, Seropédica, RJ. Fone: (21) 2682-1080, Fax (21) 2682 1120. E-mail ezonta@ufrrj.br; lanjos@ufrrj.br; gervasio@ufrrj.br; jstafanato@hotmail.com

${ }^{4}$ U niversidade Severino Sombra, Rua Dr. Fernandes Junior 89, Centro, CEP 27700-000, Vassouras RS. Fone: (21) 2471-8273. E-mail febrasil@bol.com.br
} 


\section{INTRODUÇÃO}

A região Noroeste do Estado do Rio de Janeiro vem sendo, há anos, explorada por práticas agrícolas predatórias e pelo uso inadequado do solo em áreas de relevo movimentado, de Mar de Morros. Atualmente, grande parte da região apresenta solos de baixa fertilidade e cultivados por pastagens de Brachiaria sp, em diferentes estágios de degradação, sendo comum a exposição dos solos a processos avançados de erosão de encostas e baixíssimas produtividades da pecuária de corte e leite, nos municípios da região.

A degradação de pastagens pode ser definida como a progressiva perda de produtividade e vigor das plantas, da capacidade de recuperação natural para sustentar os níveis de produção e qualidade exigida pelos animais, e da incapacidade de se superar os efeitos nocivos de pragas, doenças e invasoras (Soares Filho et al., 1992; Nascimento et al., 2006, Townsend et al., 2003). As principais causas desta degradação estão relacionadas às práticas de manejo inadequadas das plantas forrageiras e à perda gradativa da fertilidade do solo devido à exportação e à não reposição dos nutrientes (Muller et al. 2001; Boddey et al., 1993; Bortolo et al., 2001; Cecato et al., 2001; Ydoyaga et al., 2006).

Durante o processo de degradação e com a diminuição da cobertura vegetal das pastagens, ocorre um significativo aumento na competitividade pelas plantas invasoras que, em geral, são mais eficientes no uso dos escassos recursos do solo. Além disso, ocorrem perdas por lixiviação de nutrientes, como efeito de atividades agrícolas em solos naturalmente pobres ou sujeitos a baixos teores de matéria orgânica, além de perdas de ordem física, como erosão e compactação de solo (Soares Filho et al., 1992; Nascimento et al., 2006; Muller et al., 2001).

Práticas de manejo da fertilidade do solo, como a calagem, são fundamentais para o bom desempenho das pastagens e podem alterar a dinâmica de nutrientes, de forma a estabelecer um equilíbrio no sistema solo-planta-animal ou, ainda, melhorar essas relações visando à sustentabilidade.

Além da calagem, adubações de manutenção adequadas (quantitativa e qualitativamente) também são de extrema importância para o desempenho das culturas, sobretudo no Brasil, onde a prática mais comum é a utilização de espécies com baixa exigência em fertilidade, como as gramíneas do gênero Brachiaria, que são espécies mais resistentes a pragas Costa et al. (2008). Não obstante as Brachiarias serem consideradas adaptadas a condições edáficas de baixa fertilidade, são necessários estudos específicos para a introdução de novas espécies de forrageiras de maior valor nutricional e melhores produtividades de ganho de peso e produção de leite.

De acordo com Boddey et al. (1993), uma pastagem sustentável deve manter a produção animal e vegetal ao longo dos anos e ser resistente às condições climáticas; para tal, há necessidade de garantir nutrição adequada a essas plantas. Em um sistema pastoril sustentável os nutrientes minerais devem ser conservados ou repostos. Ainda, se o sistema for conduzido para alta produtividade, os nutrientes devem estar em suas formas disponíveis para serem assimilados ra- pidamente pelas plantas e garantir teores adequados, de acordo com as taxas de crescimento.

A mobilidade e a disponibilidade dos nutrientes estão ligadas às características físicas e químicas do solo e condições de manejo, sendo seu estudo imprescindível para o desenvolvimento das pastagens e a manutenção das condições de produção agrícola Bissani et al. (2004). Sendo assim, o conhecimento da dinâmica dos nutrientes no solo é fundamental, sobremaneira em áreas de relevo declivoso, onde os processos de erosão contribuem significativamente para a exportação do solo e nutrientes do sistema.

A dinâmica dos nutrientes nos solos em função da sua natureza estrutural e química, influencia diretamente sua disponibilidade para as plantas, Gilles et al. (2009). Desta forma, os estudos sobre a dinâmica dos atributos físicos, químicos e biológicos envolvidos em um sistema de pastagens se tornam cruciais para que se possa traçar as melhores técnicas a serem utilizadas na sua conservação e recuperação.

A disponibilidade de nutrientes em um sistema de manejo de pastagens degradadas deve ser investigada constantemente para se estabelecer limites e desenvolver conhecimentos específicos adaptados às condições socioeconômicas dos produtores aumentando, desta forma, a produção vegetal e melhorando a produtividade e a preservação do meio ambiente.

O objetivo deste trabalho foi avaliar a variação espaçotemporal da fertilidade do solo em uma área declivosa e cultivada com três espécies de gramíneas forrageiras, no município de Itaperuna, no Noroeste do Estado do Rio de Janeiro.

\section{MATERIAL E MÉTODOS}

O experimento foi conduzido na fazenda Pau-Ferro, dedicada à engorda de gado de corte, no município de Itaperuna, Noroeste do Estado do Rio de Janeiro, no período de 2002 a 2006. A área experimental utilizada pertencia ao projeto RADEMA, uma parceria entre a UFRRJ, através do Departamento de Solos, a PESAGRO-Rio e a EMATER-Rio, coordenado pela EMBRAPA Solos.

O clima da região apresenta características Aw, segundo Köeppen. Martorano et al. (2003) observaram indícios de que o clima na região se vem tornando mais seco e mais quente, sendo classificado como clima Megatérmico Subúmido Seco, com precipitação insuficiente em todas as estações do ano.

O solo foi classificado como Argissolo Vermelho-Amarelo Eutrófico típico, textura média/muito argilosa, A moderado, mesoeutrófico, caulinítico, mesoférrico, ácido, fase floresta tropical subcaducifólia, relevo forte ondulado EMBRAPA (2006).

Na fase inicial do projeto, em abril de 2001 (tempo zero), a área se encontrava com uma pastagem de braquiária (Brachiaria brizantha) em avançado estado de degradação. $\mathrm{O}$ experimento foi implantado na face, com maior incidência dos raios solares (face soalheira), e foi dividido em três piquetes (parcelas) com 2 ha cada um; em cada parcela foi implantada uma espécie forrageira, formando três tratamentos, em que na primeira parcela a braquiária (Brachiaria brizantha) foi recu- 
perada através de adubação corretiva, na segunda foi implantada a gramínea Tifton 85 (Cynodon spp.) e na terceira, o capim Suázi (Digitaria swazilandensis).

Os piquetes foram manejados em sistema rotacionado, com períodos de nove dias de pastejo e 19 dias de descanso. Os animais utilizados foram novilhos mestiços das raças Holandesa e Zebus, com peso médio inicial de 209 $\mathrm{kg}$. Na fase de formação dos pastos foram aplicados $80 \mathrm{~kg}$ de $\mathrm{P}_{2} \mathrm{O}_{5}$ por hectare, na forma de Superfosfato Simples. Inicialmente, a adubação de manutenção foi programada para ser dividida (início e final da época chuvosa); entretanto, por problemas operacionais o fertilizante foi aplicado uma única vez no ano. No primeiro ano foi aplicado em fevereiro; nos anos seguintes, sempre entre abril e maio, logo após um período de chuva. No primeiro ano (2001) o adubo utilizado foi a fórmula 25-05-20 e nos anos seguintes, a fórmula utilizada foi 20-05-20, quando se aplicaram $200 \mathrm{~kg}$ das formulações por ha.

O pastejo monitorado se iniciou em 13/01/2003 e a carga animal nos piquetes foi de 3,25; 2,69 e 3,63 animal por hectare para as gramíneas Braquiária, Suázi e Tifton 85 , respectivamente.

Realizaram-se nove datas de coleta, a saber: a primeira em abril de 2002, seguida das coletas de julho de 2002, maio de 2003, novembro 2003, maio de 2004, agosto de 2004, maio de 2005 e agosto de 2005. A última coleta foi realizada em maio de 2006; nesta sequência se procurou avaliar os parâmetros de fertilidade durante as estações de seca e chuva (inverno e verão).

As coletas foram feitas em três pontos do piquete, seguindo-se sempre os terços inferior, médio e superior da encosta, de maneira a acompanhar o efeito da declividade. As amostras foram coletadas em trincheiras, no total de três trincheiras por parcela e nove pontos de coleta, dentro da área experimental. Dentro das trincheiras foram realizadas, em uma de suas paredes, coletas de solo com duas repetições (R1 e R2), utilizando-se monolitos metálicos de $1 \mathrm{dm}^{3}$; obtiveram-se amostras nas profundidades de $0-0,1,0,1-0,2,0,2-0,3-0,3-0,4$ 0,4-0,5, 0,5-0,6 e 0,6-0,7 m, além de 14 amostras por trincheira, 42 por parcela cujo total nos três tratamentos em cada coleta foi de 126 amostras.

Utilizaram-se $100 \mathrm{~g}$ TFSA, para as análises químicas e determinação de $\mathrm{pH}$ em água, $\mathrm{Mg}, \mathrm{Ca}, \mathrm{Al}, \mathrm{P}, \mathrm{Na}, \mathrm{K}$ e carbono orgânico, de acordo com EMBRAPA (1997).

O experimento foi composto de um fatorial complexo e incompleto, sendo necessário hierarquizar os fatores, visandose à melhor compreensão do comportamento dos parâmetros avaliados. A partir da hierarquização se aplicaram modelos distintos em função do tipo de dado obtido, desde a análise de variância até modelos multivariados.

Para este estudo foram feitos análises de variância e teste de diferenças entre médias (Scott-Knott, $\mathrm{P}<0,05$ ), com o programa SAEG®. Após esta análise os dados foram comparados por análise de componentes principais (PCA); esta análise de componentes principais (PCA), foi feita utilizandose o programa $\mathrm{Xl}$ Stat ${ }^{\circledR}$, visando principalmente ao agrupamento desses parâmetros de fertilidade; instalaram-se, também, técnicas de estatística para análises de correlação de Pearson, dos parâmetros de fertilidade.

\section{RESULTADOS E DISCUSSÃO}

As análises das amostras de solo do tempo zero (abril de 2001) apresentaram teores de nitrogênio $(\mathrm{N})$ variando entre 0,5 a $1,8 \mathrm{~g} \mathrm{~kg}^{-1}$, estando as médias em torno de $1,05 \mathrm{~g} \mathrm{~kg}^{-1}$, com os maiores níveis na camada superficial. Para o carbono orgânico os valores estiveram entre 7,02 e 17,3 $\mathrm{g} \mathrm{kg}^{-1}$ (média de $\left.9 \mathrm{~g} \mathrm{~kg}^{-1}\right)$, também maiores na camada superficial $(0-10 \mathrm{~cm})$. Entre as posições da encosta, o terço superior apresentou os maiores teores de C.Org., o que pode ser relacionado com a maior intensidade dos processos erosivos dos demais pontos de observação, principalmente no terço médio da encosta.

A acidez potencial $(\mathrm{H}+\mathrm{Al})$ apresentou comportamento semelhante ao do C.Org. Quanto ao $\mathrm{pH}$, os valores foram moderadamente ácidos (pH entre 5,4 - 6,5), não se observando grandes variações na encosta nem entre profundidades. Os níveis de cálcio $(\mathrm{Ca})$ foram classificados médios, com pequenas variações em profundidade. Os maiores teores de Ca foram verificados nos terços médio e inferior de encosta. $\mathrm{O}$ magnésio (Mg) apresentou o mesmo comportamento do $\mathrm{Ca}$, com níveis na classe baixo-média. De maneira geral, os teores de alumínio ( $\mathrm{Al}$ ) foram baixos ou muito baixos. Quanto ao fósforo $(\mathrm{P})$, os teores foram baixos em todas as seções da encosta; já para o potássio (K), os níveis foram muito baixos, com valores médios de $0,03 \mathrm{cmol}_{\mathrm{c}} \mathrm{kg}^{-1}$ e pequena variação em profundidade.

Nas demais amostragens (2002 a 2006), os valores de $\mathrm{pH}$, $\mathrm{Al}, \mathrm{H}+\mathrm{Al}, \mathrm{Ca}, \mathrm{Mg}, \mathrm{P}, \mathrm{K}, \mathrm{C}$.Org, V\%, valor S e valor T, demonstraram pouca variação dentro dos tratamentos, com poucas diferenças significativas $(\mathrm{P}>0,05) \mathrm{em}$ profundidade $(0,0-0,7$ $\mathrm{m}$ ) entre tratamentos, nos meses em que foram realizadas as coletas. Desta forma e se considerando o número muito grande de tabelas e a inexpressão das diferenças obtidas pela comparação de médias, optou-se por apresentar os dados brutos da Tabela 1, haja vista serem observadas pequenas diferenças em algumas profundidades entre as áreas dos tratamentos e ao longo do tempo. Na Tabela 1 se apresentam os dados das médias de todas as coletas e parâmetros de fertilidade do solo analisados durante todo o período experimental, de 2002 a 2006, entre os diferentes tratamentos.

A similaridade entre os valores observados demonstra uma semelhança na fertilidade do solo entre as áreas cultivadas pelas três espécies forrageiras, entre as profundidades $(0,0-$ $0,7 \mathrm{~m})$, e na maior parte das coletas realizadas na área experimental. Os valores que apresentaram as diferenças mais sensíveis foram os do fósforo (P).

Após a interpretação desses dados, realizou-se a análise de componentes principais (PCA), para o agrupamento e comparação dos parâmetros de fertilidade analisados. Para esta análise foram selecionados apenas os principais parâmetros significativos determinados pela matriz de correlação de Pearson, sendo: $(\mathrm{Mg}),(\mathrm{Ca}+\mathrm{Mg}), \mathrm{Al}, \mathrm{K},(\mathrm{H}+\mathrm{Al})$, carbono orgânico e os valores de $\mathrm{S}, \mathrm{T}$ e $\mathrm{V} \%$.

Considerou-se, nesta análise de componentes principais (PCA), a proporcionalidade do comprimento de cada linha com a sua importância, sendo os ângulos entre elas um reflexo das intercorrelações entre as variáveis, Fontana et al. (2008) ana- 
Tabela 1. Média dos valores de $\mathrm{pH}, \mathrm{Al}, \mathrm{H}+\mathrm{Al}, \mathrm{Ca}, \mathrm{Mg}, \mathrm{Ca}+\mathrm{Mg}$, $K$, valor $T$ e valor $S$ expressa em $\mathrm{cmolc} \mathrm{dm}^{-3}, \mathrm{~V} \%, \mathrm{P}$ com valores médios em $\mathrm{mg} \mathrm{kg}^{-1} \mathrm{e} C .0 \mathrm{rg}$ com valores médios expressos em $\mathrm{g} \mathrm{kg}^{-1}$, entre abril de 2002 a maio de 2006, nas profundidades $(0-0,7 \mathrm{~m})$

\begin{tabular}{|c|c|c|c|c|c|c|c|c|c|}
\hline \multirow{2}{*}{ Profundidade (m) } & \multicolumn{3}{|c|}{ Braquiaria } & \multicolumn{3}{|c|}{ Tifton } & \multicolumn{3}{|c|}{ Suazi } \\
\hline & $\mathrm{pH}$ & Al & $\mathrm{H}+\mathrm{Al}$ & $\overline{\mathrm{pH}}$ & Al & $\mathrm{H}+\mathrm{Al}$ & $\overline{\mathrm{pH}}$ & Al & $\mathrm{H}+\mathrm{Al}$ \\
\hline $0,0-0,1$ & 5,6 & 0,0 & 3,3 & 5,6 & 0,1 & 2,9 & 5,6 & 0,1 & 2,8 \\
\hline $0,1-0,2$ & 5,5 & 0,2 & 3,1 & 5,6 & 0,1 & 2,3 & 5,7 & 0,0 & 2,6 \\
\hline $0,2-0,3$ & 5,5 & 0,2 & 3,1 & 5,6 & 0,1 & 2,2 & 5,6 & 0,1 & 2,5 \\
\hline $0,3-0,4$ & 5,4 & 0,3 & 3,1 & 5,6 & 0,1 & 2,2 & 5,6 & 0,1 & 2,3 \\
\hline $0,4-0,5$ & 5,3 & 0,3 & 3,4 & 5,5 & 0,1 & 2,5 & 5,6 & 0,1 & 2,7 \\
\hline $0,5-0,6$ & 5,3 & 0,3 & 3,5 & 5,5 & 0,2 & 2,5 & 5,6 & 0,1 & 2,4 \\
\hline \multirow[t]{2}{*}{$0,6-0,7$} & 5,6 & 0,3 & 3,8 & 5,5 & 0,2 & 2,7 & 5,6 & 0,2 & 2,5 \\
\hline & $\mathrm{Ca}$ & $\mathrm{Mg}$ & $\mathrm{Ca}+\mathrm{Mg}$ & $\mathrm{Ca}$ & $\mathrm{Mg}$ & $\mathrm{Ca}+\mathrm{Mg}$ & $\mathrm{Ca}$ & $\mathrm{Mg}$ & $\mathrm{Ca}+\mathrm{Mg}$ \\
\hline $0,0-0,1$ & 1,5 & 1,5 & 3,0 & 1,6 & 1,7 & 3,2 & 1,7 & 1,6 & 3,3 \\
\hline $0,1-0,2$ & 1,3 & 1,1 & 2,4 & 1,5 & 1,3 & 2,9 & 1,7 & 1,4 & 3,0 \\
\hline $0,2-0,3$ & 1,2 & 1,0 & 2,2 & 1,3 & 1,3 & 2,6 & 1,4 & 1,3 & 2,6 \\
\hline $0,3-0,4$ & 1,2 & 0,9 & 2,0 & 1,2 & 1,2 & 2,4 & 1,3 & 1,1 & 2,4 \\
\hline $0,4-0,5$ & 1,1 & 1,1 & 2,1 & 1,1 & 1,0 & 2,2 & 1,3 & 1,0 & 2,3 \\
\hline $0,5-0,6$ & 1,0 & 1,0 & 2,0 & 1,2 & 1,1 & 2,2 & 1,3 & 1,1 & 2,3 \\
\hline \multirow[t]{2}{*}{$0,6-0,7$} & 0,8 & 1,1 & 1,9 & 1,1 & 1,1 & 2,1 & 1,2 & 1,0 & 2,2 \\
\hline & $P$ & $\mathrm{~K}$ & C.Org & $P$ & $\mathrm{~K}$ & C.Org & $P$ & $K$ & C.Org \\
\hline $0,0-0,1$ & 7,5 & 0,2 & 13,3 & 2,7 & 0,2 & 13,2 & 5,2 & 0,3 & 13,6 \\
\hline $0,1-0,2$ & 2,1 & 0,1 & 11,0 & 1,6 & 0,1 & 10,7 & 4,1 & 0,1 & 10,6 \\
\hline $0,2-0,3$ & 1,8 & 0,1 & 8,8 & 1,3 & 0,1 & 8,9 & 2,5 & 0,1 & 8,9 \\
\hline $0,3-0,4$ & 1,5 & 0,1 & 7,9 & 1,1 & 0,0 & 8,4 & 3,1 & 0,1 & 8,2 \\
\hline $0,4-0,5$ & 2,6 & 0,0 & 7,4 & 4,0 & 0,0 & 7,1 & 0,9 & 0,1 & 7,4 \\
\hline $0,5-0,6$ & 1,7 & 0,0 & 6,5 & 1,0 & 0,0 & 6,5 & 3,6 & 0,0 & 6,8 \\
\hline \multirow[t]{2}{*}{$0,6-0,7$} & 4,0 & 0,0 & 6,1 & 2,7 & 0,0 & 6,6 & 1,0 & 0,0 & 6,2 \\
\hline & $S$ & $\mathrm{~T}$ & V\% & $S$ & $\mathrm{~T}$ & V\% & $S$ & $\mathrm{~T}$ & V\% \\
\hline $0,0-0,1$ & 3,2 & 6,5 & 57,4 & 3,5 & 6,4 & 57,3 & 3,6 & 6,5 & 60,1 \\
\hline $0,1-0,2$ & 2,6 & 5,7 & 55,1 & 2,9 & 5,2 & 59,8 & 3,1 & 5,7 & 58,9 \\
\hline $0,2-0,3$ & 2,3 & 5,4 & 54,0 & 2,7 & 4,8 & 60,0 & 2,7 & 5,2 & 56,4 \\
\hline $0,3-0,4$ & 2,1 & 5,2 & 52,6 & 2,5 & 4,7 & 58,8 & 2,5 & 4,8 & 56,5 \\
\hline $0,4-0,5$ & 2,2 & 5,6 & 50,9 & 2,2 & 4,7 & 55,1 & 2,4 & 5,0 & 49,8 \\
\hline $0,5-0,6$ & 2,1 & 5,5 & 49,5 & 2,3 & 4,8 & 54,8 & 2,4 & 4,8 & 54,1 \\
\hline $0,6-0,7$ & 2,0 & 5,8 & 47,6 & 2,2 & 4,9 & 52,3 & 2,2 & 4,7 & 52,9 \\
\hline
\end{tabular}

lisadas. O ângulo entre linhas e o eixo de ordenação representam certo grau de correlação.

Os dados avaliados foram dispostos em dois eixos, $\mathrm{F} 1 \mathrm{e}$ $\mathrm{F} 2$, nos quais foram ordenados os valores de $\mathrm{Ca}+\mathrm{Mg}, \mathrm{K}, \mathrm{S}$, C.org, T, H+Al, Mg, V\% e Al (Figura 1).

As avaliações feitas pela PCA nas áreas dos tratamentos da Fazenda Pau Ferro, demonstram que ocorrem cinco agrupamentos principais que merecem destaque nas variações dos dados, quando se avaliam os parâmetros de fertilidade e sua variabilidade nas áreas (Figura 2).

Os agrupamentos observados na dispersão dos fatores 1 e 2, foram: (a) influenciados pelos $\mathrm{Al}, \mathrm{H}+\mathrm{Al}$ e valor $\mathrm{T}$; (b) e (c) influenciados por outros fatores não correlacionados pela PCA; (d) influenciados pelos $\mathrm{Ca}+\mathrm{Mg}, \mathrm{C}$.org, $\mathrm{K}$ e valor $\mathrm{S}$ e o agrupamento (e) que, nos dados, foram influenciados pelo magnésio $\mathrm{Mg}$ e pelo valor V (Figura 2).

Todas as áreas dos tratamentos demonstraram uma semelhança entre a maior parte das dispersões, apresentando-se distribuídas de forma idêntica entre os diversos parâmetros avaliados; no entanto, o (a) demonstra um agrupamento mai-

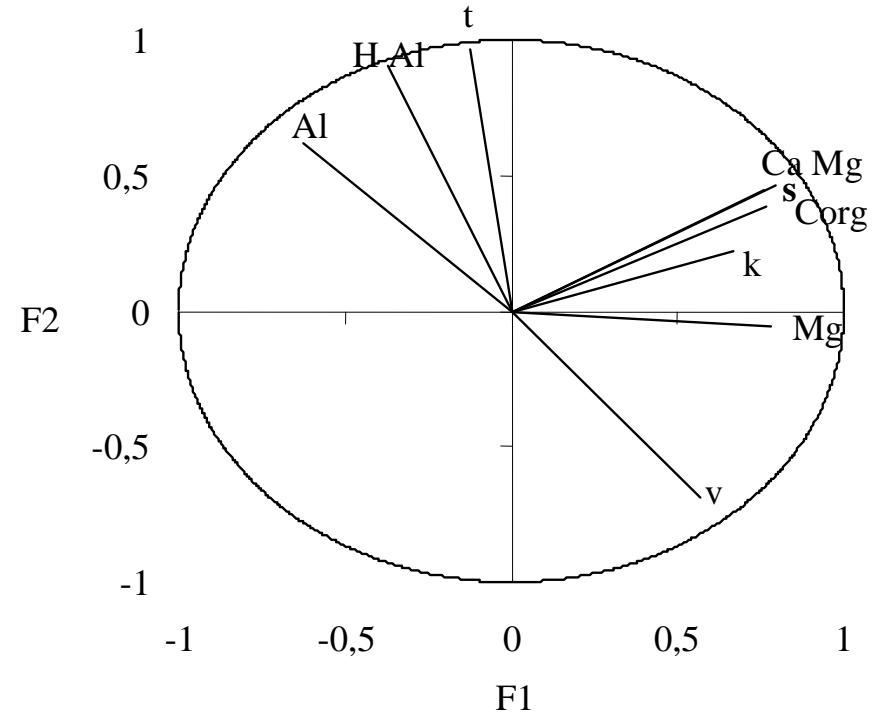

Figura 1. Distribuição dos parâmetros de fertilidade da Fazenda Pau Ferro, Itaperuna, RJ, avaliados pela análise dos componentes principais (PCA)

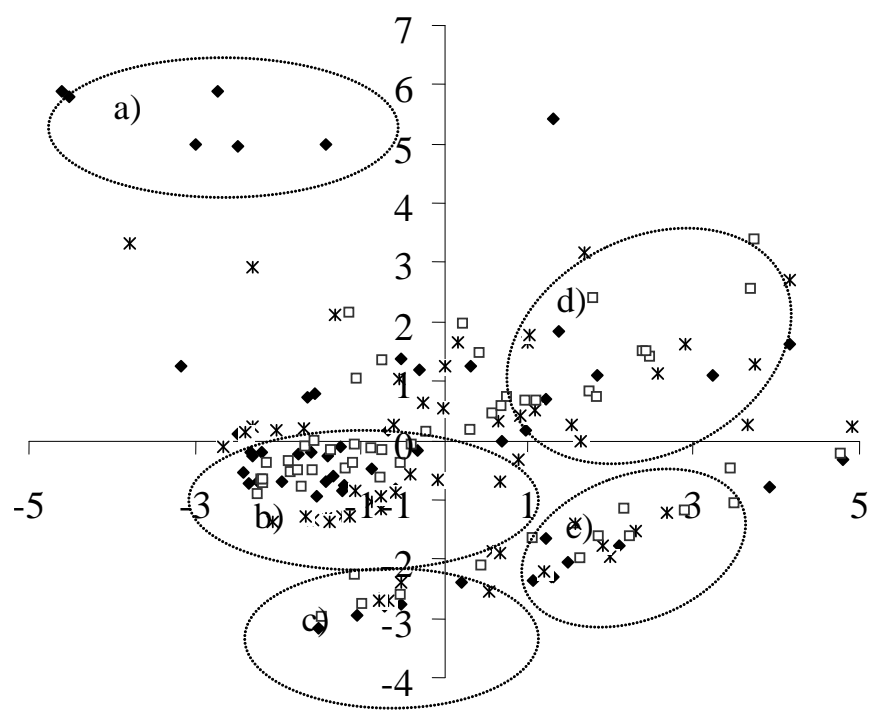

Braquiária * Tifton 85 ¿ Suázi

Figura 2. Dispersão dos escores dos fatores 1 e 2 eáreas dos tratamentos estudados na fazenda Pau Ferro, Itaperuna, RJ, avaliados pela análise dos componentes principais (PCA). a b c d e, principais agrupamentos

or das variâncias da área do tratamento Brachiaria brizantha, o que indica uma pequena variação desta área em relação aos demais tratamentos (Figura 2). Este fato pode ser complementado pelas diferenças observadas nos valores de alumínio, $\mathrm{H}+\mathrm{Al}$ e do valor T da área do tratamento Brachiaria brizanta, que apresentaram algumas pequenas diferenças entre as demais áreas em algumas épocas de coleta (Tabela 1).

No agrupamento (d) também se pode observar um número maior de variâncias em relação à área do tratamento Digitaria swazilandensis com maior acúmulo dessas variâncias na parte inferior esquerda do campo, assinalado para o agrupamento (d) (Figura 2).

Esta variação também pode ser visualizada nos dados da análise de variância, em que os valores de $\mathrm{V} \%$, valor $\mathrm{S}$ e 
$\mathrm{Ca}+\mathrm{Mg}$, apresentam diferenças significativas, explicando a variação desses parâmetros encontrados na área do tratamento Digitaria swazilandensis que, em geral, foram maiores do que os valores encontrados nas áreas dos demais tratamentos e observados em coletas realizadas nos períodos de abril 2002 maio de 2003 e, em alguns casos em maio de 2006.

Em uma análise complementar dos resultados de fertilidade obtidos no experimento (Tabela 1), utilizaram-se, como referência para efeito comparativo, as tabelas de interpretação de resultados de análises de rotina de dados obtidos de amostra de solo para o Estado do Rio de Janeiro, Almeida et al. (1988). Os parâmetros de fertilidade comparados foram: $\mathrm{pH}$, $\mathrm{P}, \mathrm{K}, \mathrm{Al}, \mathrm{Ca}+\mathrm{Mg}$, e Carbono orgânico do solo (C. Org), comumente utilizados nas tabelas de Interpretação dos resultados de análises de rotina no estado do Rio de Janeiro.

Quanto aos níveis de $\mathrm{pH}$ do solo, variaram entre 5,3 e 6,5 na área experimental, sendo esta faixa considerada, de acordo com os valores, entre fortemente ácida e moderadamente ácida, 4,4-5,3 e 5,4-6,5 respectivamente, Almeida et al. (1988).

Os valores médios tenderam a variar, principalmente entre 5,3 e 5,7 (Tabela 1), tendo a maior parte das áreas dos tratamentos mantido esta variação.

Esses valores diferem um pouco dos encontrados inicialmente na área (Tempo zero); no entanto, se considerados os valores médios observados, pode-se dizer que eles mantêm a mesma classificação encontrada no período inicial de projeto, quando se avaliam todas as profundidades estudadas; isto ocorreu provavelmente em função do manejo realizado na área, sobretudo devido às adubações. Segundo demonstraram Schlindwein \&. Anghinoni (2000), os valores de nutrientes no solo e do pH podem variar em função do manejo adotado e do método de coleta do solo utilizado sendo, também, influenciados por fatores outros, como técnicas de manejo do solo e adição de fertilizantes (Oliveira, 2003a.).

A faixa de $\mathrm{pH}$ encontrada na área dos tratamentos se encontra abaixo de 6,0 - 6,5 que, segundo Malavolta (1980), é uma faixa de $\mathrm{pH}$ da qual se observa a disponibilidade máxima de muitos elementos sendo que, para outros, a disponibilidade não é limitante caindo também a disponibilidade de $\mathrm{Al}$, no entanto, os dados de $\mathrm{pH}$ nas áreas dos tratamentos demonstram que muitos dos valores observados se mantêm próximos aos valores desta faixa $(6,0-6,5)$, garantindo boas disponibilidades de elementos importantes para a nutrição vegetal, como o P, K e o N.

Quanto aos níveis de alumínio, os valores variaram entre as coletas, ocorrendo diminuição ao longo do período de estudo de forma que, ao final das coletas, o mesmo não foi detectado mas ao longo do estudo os níveis de alumínio no solo indicaram, em sua maioria, valores oscilando entre 0,0 e $0,3 \mathrm{cmol}_{\mathrm{c}} \mathrm{dm}^{-}$ 3 , predominando os valores médios de $0,1,0,2$, e $0,3 \mathrm{cmol}_{\mathrm{c}} \mathrm{dm}^{-}$ 3 em profundidade, principalmente nas profundidades abaixo de 0,3 m (Tabela 1). Desta forma, pode-se considerar os valores de alumínio $(\mathrm{Al})$ na área experimental como baixos e equivalentes entre as diversas áreas dos tratamentos.

Os níveis de alumínio podem comprometer o crescimento vegetal, afetando o crescimento radicular e influenciando, de diversas formas, na absorção e disponibilidade de nutrientes sendo, por isso, um dos fatores que mais limitam o desenvol- vimento das plantas (Silva et al., 2004). Logo, a manutenção de baixos níveis ao longo do tempo, pode garantir a produtividade das pastagens. Os níveis de $\mathrm{Al}$ na área podem ter sido mantidos baixos em função da faixa de $\mathrm{pH}$ observada, e a forma de manejo pode influenciar a acidez e a disponibilidade de alumínio. O que inclui, também, características como o aporte de resíduos vegetais, influenciado pelo manejo adotado na área, o que pode ter auxiliado na manutenção dos níveis de $\mathrm{pH}$ (Nascimento et. al., 2003).

Quanto aos níveis de $\mathrm{Ca}+\mathrm{Mg}$ na área dos tratamentos, oscilaram entre 1,9 e $3,3 \mathrm{cmol}_{\mathrm{c}} \mathrm{dm}^{-3}$, porém os valores médios estiveram em torno de entre 2,0 e $3,3 \mathrm{cmol}_{\mathrm{c}} \mathrm{dm}^{-3}$ (Tabela 1); sendo assim, os níveis de $\mathrm{Ca}+\mathrm{Mg}$ podem ser classificados como de teores médios, o que demonstra que houve manutenção nos níveis de $\mathrm{Ca}+\mathrm{Mg}$ na área visto que, quando comparados com os valores encontrados no início do projeto, eles se mantiveram semelhantes e dentro da mesma faixa de classificação; esta avaliação indica que as adubações e o manejo na área experimental foram eficazes em manter os níveis de bases na área, pois os cátions do solo, como $\mathrm{Na}^{+}, \mathrm{K}^{+}, \mathrm{Ca}^{2+}$, $\mathrm{Mg}^{2+}$ podem ser levados pela ação das chuvas ou serem lixiviados; desta forma, a prática adotada e a manutenção correta dos níveis de fertilidade favoreceram a manutenção desses nutrientes no solo e nas plantas, (Oliveira et al., 2003b).

Quanto aos parâmetros que refletem a acidez no solo, como $\mathrm{pH}, \mathrm{Al}$ e $\mathrm{Ca}+\mathrm{Mg}$, pode-se dizer que se mantiveram estáveis e foram semelhantes entre as áreas dos tratamentos; aparentemente, não houve perda ou diminuição que indicasse um avanço no processo de degradação, o que reflete em um indicativo da possibilidade de sustentabilidade do sistema de pastagens na área; já que se deve levar em conta que a perda de nutrientes e a acidificação são processos de degradação do solo; essas perdas comprometem a produtividade e a sustentabilidade dos vegetais em áreas de pastagens (Oliveira et al. (2005; 2003a.).

Quanto aos níveis de $\mathrm{K}$ nas áreas dos tratamentos da fazenda Pau Ferro, os valores médios variaram entre 0,0 e 0,3 $\mathrm{cmol}_{\mathrm{c}} \mathrm{dm}^{-3}$ (Tabela 1); em sua maioria os níveis tenderam a permanecer abaixo de $0,3 \mathrm{cmol}_{\mathrm{c}} \mathrm{dm}^{-3}$; nas profundidades abaixo de 0-30 cm, os valores permaneceram inferiores a $0,2 \mathrm{cmol}_{\mathrm{c}}$ $\mathrm{dm}^{-3}$ e os valores médios mais constantes oscilaram entre 0,1 e 0,0 (7,9 e 39 ppm) (Tabela 1), considerados baixos, na maior parte dos dados avaliados (Almeida et al., 1988).

Os níveis de fósforo encontrados nas áreas dos tratamentos foram sensivelmente mais diferenciados entre as áreas, quando comparados com os tratamentos. Os valores médios oscilaram entre $1,5 \mathrm{a} 7,5 \mathrm{mg} \mathrm{kg}^{-1}$ para a área do tratamento Braquiária, 1,0 a 4,0 $\mathrm{mg} \mathrm{kg}^{-1}$ para a área do tratamento Tifton 85 e 0,9 a 5,2 $\mathrm{mg} \mathrm{kg}^{-1}$ para a área do tratamento Suázi (Tabela 1). Desta forma, os níveis de $P$ avaliados podem ser considerados baixos para todas as áreas (Almeida et al., 1988).

Em referência ao carbono orgânico (C.Org) no solo, os dados não demonstraram variação significativa ao longo das coletas realizadas e os valores variaram entre 5,3 e $16,5^{\circ} \mathrm{g} \mathrm{kg}-$ 1; entretanto, na maior parte dos dados analisados os valores de C. org. se apresentaram abaixo de $10 \mathrm{~g} \mathrm{~kg}^{-1}$ e com valores médios oscilando entre 6,1 e $13,6 \mathrm{~g} \mathrm{~kg}^{-1}$ entre as áreas dos tratamentos (Tabela 1), classificados baixos. 
Em relação ao nível inicialmente encontrado nas áreas pode se observar, nos níveis de carbono orgânico (C. org) no solo, diminuição dos valores em relação ao tempo zero, porém os valores de carbono orgânico (C. org) no solo não apresentaram variação significativa no período de estudo, principalmente quando se avalia a camada de $0-0,3 \mathrm{~m}$.

Esses resultados podem estar demonstrando um provável efeito do manejo inicial, pois áreas de pastagens podem apresentar decréscimo nos níveis de carbono orgânico ou, então, não apresentar diferenças nos seus níveis, ao longo de alguns anos (Lilienfein et al., 2003; Oliveira et al., 2005).

Então, pode-se constatar que o sistema pastoril implantado na área da Fazenda Pau Ferro contribuiu para a manutenção dos níveis de carbono orgânico no solo (C. org), quando então a mudança no regime de corte e no sistema de pastejo pode causar crescimento diferenciado nas plantas influenciando, assim, a dinâmica do crescimento vegetal, de forma a favorecer a manutenção da parte aérea da pastagem e do sistema radicular, levando, assim, a uma manutenção melhor nos níveis de C. org no solo (Pinto et al., 2001).

Cordeiro (2008) demonstrou, em estudo nesta mesma área, realizado de agosto de 2004 a agosto de 2005, que atributos como a distribuição do peso de agregados em função das classes de tamanho, em especial na classe $>2,0 \mathrm{~mm}$, e o teor de carbono orgânico, foram indicadores sensíveis aos efeitos dos tratamentos, tipos de gramínea e sazonalidade.

Desta forma e se levando em conta o fato de que as características morfogênicas do relvado são responsáveis pelas variações que ocorrem na estrutura de pastagem, Pinto et al. (2001), e que a degradação de pastagens pode ser caracterizada por um estoque menor de carbono do compartimento de resíduos vegetais, pode-se concluir que o nível de carbono orgânico (C. org) encontrado nas áreas dos tratamentos refletiu o seu manejo sendo, portanto, a mudança no sistema de desfolha, mantida pela rotação dos animais nas áreas dos tratamentos, um dos modos de influenciar os níveis de carbono orgânico no solo, ao longo do período de estudo.

Uma análise global dos dados de fertilidade do solo obtidos na área experimental, permite sugerir que, na maior parte das coletas, os dados não apresentaram diferenças que pudessem determinar maior nível de fertilidade no aspecto químico de uma área em relação à outra, sendo a maior diferença observada para o fósforo.

As diferenças nos parâmetros de fertilidade avaliados em profundidade, encontradas entre as áreas em algumas épocas de coleta, podem estar relacionadas ao fato das áreas apresentarem diferenças em função de características próprias. As pequenas diferenças na topografia e no ponto de coleta podem ter causado essas variações, pois o solo é formado a partir de materiais de origem passíveis de variar em função da posição do relevo para dado clima e vegetação influenciando, assim, a distribuição de nutrientes.

\section{CONCLUSÕES}

1. As adubações e o manejo das pastagens nas áreas dos tratamentos foram eficientes na manutenção das condições químicas no ambiente edáfico podendo, então, diminuir a velocidade no processo de degradação do solo na área.

2. As áreas dos tratamentos, Braquiária (Brachiaria brizantha), Tifton 85 (Cynodon spp.) e do capim Suázi (Digitaria swazilandensis), se apresentaram semelhantes, no que se refere aos atributos de fertilidade do solo, podendo ser a pouca variação encontrada na área ao longo dos anos de estudo, um indicativo da eficiência do manejo rotacionado adotado na distribuição dos nutrientes e na manutenção dos níveis de fertilidade na área.

\section{LITERATURA CITADA}

Almeida, D. L; Freire, R. L.; Cunha, L. H.; Santos, G. A.; Sobrinho, N. M. B. A.; Eira, P. A. Fertilidade do solo In: De Polli, H; Araújo G. S.; Cunha, L. H; Freire, R., L.; Sobrinho, N. M. B. A.; Pereira N. N. C.; Eira P. A. da; Bloise, M. R.; Salek, R. C. Manual de adubação para o Estado do Rio de Janeiro. Itaguaí: UFRRJ, n.2, 1988, p.13-23. Série Ciências Agrárias

Bissani, C. A.; Gianello, C.; Tedesco M. J. Camargo, F. A. O. Fertilidade dos solos e manejo de adubação de culturas. Porto Alegre: Metrópole Indústria Gráfica, 2004. 322p.

Boddey, R. M.; Resende, C. P. Schunke, R. M. Sustentabilidade de pastagens consorciadas e de gramíneas em monocultura: o papel chave das transformações de nitrogênio, In: 30a Reunião anual da Sociedade Brasileira de Zootecnia, 1993, Rio de Janeiro. Anais... Rio de Janeiro: SBZ, 1993. p.141-173.

Bortolo, M.; Cecato, U.; Martins, E. N.; Cano, C. C. P.; Coalho M. R.; Canto, M. W. : Santos G. T. Avaliação de uma pastagem de coastcross-1 (Cynodon dactylon (L.) Pers) sob diferentes níveis de matéria seca residual. Revista Brasileira de Zootecnia, v.30, n.3, p.627-635, 2001.

Cecato U.; Castro C. R. C.; Canto, M. W.; Peternelli M.; A. Júnior, J.; Jobim, C. C.; Cano, C. C. P. Perdas de forragem em capim-tanzânia (Panicum maximum Jacq cv. Tanzania-1) manejado sob diferentes alturas sob pastejo. Revista Brasileira de Zootecnia, v.30, n.2, p.295-301, 2001

Cordeiro, F. C.; Pereira, M. G.; Anjos L. H. C.; Stafanato J. B.; Zonta E. Avaliação de atributos edáficos em áreas de pastagens em relevo movimentado em Itaperuna-RJ. Caatinga, v.21, n.3, p.179-191, 2008.

Costa, C.; Meirelles, P. R. L.; Silva, J. J.; Factori, M. A. Evolução das pastagens cultivadas e do efeito bovino no Brasil. Revista Veterinária e Zootecnia, v.15, n.1, p. 8-17, 2008.

EMBRAPA - Empresa Brasileira de Pesquisa Agropecuária. Manual de métodos de análise de solo. Rio de Janeiro: Embrapa CNPS, 1997. 212p.

EMBRAPA - Empresa Brasileira de Pesquisa Agropecuária. Centro Nacional e Pesquisa em Solos. Sistema Brasileiro de Classificação de Solos. Brasília: Embrapa-SPI; Rio de Janeiro: Embrapa-Solos, 2006. 306p.

Fontana, A.; Pereira, M. G.; Anjos, L. H. C.; Benites, V. M. Distribution of Organic Carbon in the Humic Fractions of Diagnostic Horizons from Brazilian Soils. Communications in Soil Science and Plant-Analysis, v.39, n.7, p.951 971, 2008. 
Gilles L.; Cogo N. P.; Bissani C. A.; Bagatini T.; Portela J. C. Perdas de água, solo, matéria orgânica e nutriente por erosão hídrica na cultura do milho implantada em área de campo nativo, influenciadas por métodos de preparo do solo e tipos de adubação. Revista. Brasileira de Ciência do Solo, v.33, n.5, p.1427-1440, 2009.

Lilienfein, J.; Wilcke, W.; Vilela, L.; Ayarza, M. A.; Lima, S. C.; Zech, W. Soil fertility under native Cerrado and pasture in the Brazilian savanna. Soil Scienci Society of America Journal, v.67, p.1195-1205, 2003.

Malavolta, E. Elementos de nutrição mineral de plantas, São Paulo: Agronômica Ceres Ltda., 1980, 251p.

Martorano, L. G.; Rossiello, R. P. O.; Meneguelli, N. A.; Lumbreras, J. F.; Leonidas, S. S. V.; Motta, P. E. F.; Rebello, E. R. G.; Martins, G. S. Aspectos climáticos do noroeste fluminense, RJ. Rio de Janeiro: Embrapa Solos, 2003. 26p.

Muller, M. M. L.; Guimarães, M. de F.; Desjardins,T.; Silva, M. P. F. da. Degradação de pastagens na Região Amazônica:propriedades físicas do solo e crescimento de raízes. Pesquisa Agropecuária Brasileira, v.36, n.11, p.14091418, 2001.

Nascimento, J. T.; Silva, I. F.; Santiago, R. D.; Silva Neto, L. F. Efeito de leguminosas nas características químicas e matéria orgânica de um solo degradado. Revista Brasileira de Engenharia Agrícola e Ambiental, v.7, n.3, p.457-462, 2003.

Nascimento, M. C.; Riva, R. D. D.; Chagas, C. S.; Oliveira, H.; Dias, L. E.; Fernandes Filho, E. I.; Soares, V. P. Uso de imagens do sensor ASTER na identificação de níveis de degradação em pastagens. Revista Brasileira de Engenharia Agrícola e Ambiental, v.10, n.1, p.196-202, 2006.

Oliveira P. P. A.; Boaretto, A. E.; Trivelin,P. C. O.; Oliveira W. S.de; Corsi M. Liming and fertilization to restore degraded Brachiaria decumbens pastures grown on an entisol. Scientia Agrícola, v.60, n.1, p.125-131, 2003a.
Oliveira P. P. A.; Ocheuze P. C. Trivelin, Oliveira W. S.; Corsi M. Fertilização com $\mathrm{N}$ e S na recuperação de pastagem de $B r a-$ chiaria brizantha cv. marandu em Neossolo Quartzarênico. Revista Brasileira de Zootecnia, v.34, n.4, p.1121-1129, 2005.

Oliveira, P. P. A.; Trivelin, P. C. O.; Oliveira, W. S. Eficiência da fertilização nitrogenada com uréia (15 n) em Brachiaria brizantha cv. Marandu associada ao parcelamento de superfosfato simples e cloreto de potássio. Revista Brasileira de Ciência do Solo, n.27, p.613-620, 2003b.

Pinto, L. F. M.; Silva, S. C. ; Sabrissia, A. F.; Carvalho, C. A. B.; Carnevalli, R. A.; Fagundes, J. L.; Pedreira, C. G. Dinâmica do acúmulo de matéria seca em pastagens de tifton 85 sob pastejo. Scientia Agrícola, v.58, n.3, p.439-447. 2001.

Schlindwein, J. A.; Anghinoni, I. Variabilidade horizontal de atributos de fertilidade e amostragem do solo no sistema plantio direto. Revista Brasileira de Ciência do Solo, n. 24, p.85-91. 2000.

Silva, M. C.; Santos, M. V. F.; D. Júnior, J. C. B.; Lira, M. A.; Santana, D. F. Y.; Farias, I.; Santos, V. F. Avaliação de métodos para recuperação de pastagens de Braquiária no agreste de Pernambuco. Revista Brasileira de Zootecnia, v.33, n.6, p.1999-2006, 2004.

Soares Filho, C. V.; Monteiro F. A.; Corsi, M. Recuperação de pastagens degradadas de Brachiaria decumbens . 1. Efeito de diferentes tratamentos de fertilização e manejo. Pasturas Tropicales, v.14, n.2, p.2-6, 1992.

Townsend, C. R. N.; Costa, L.; Mendes, A. M.; Resende, L. A. D.; Pereira, R. G. A. Resistência à penetração do solo sob pastagens degradadas na Amazônia Ocidental, submetidos a diferentes métodos de introdução de leguminosas forrageiras. Pasturas Tropicales, v.25, n.3, p.36-38, 2003.

Ydoyaga, D. F.; Lira, M. A.; Santos, M. V. F.; Júnior, J. C. B. D.; Silva, M. C.; Santos, V. F.; Fernandes, A. P. M. Métodos de recuperação de pastagens de Brachiaria decumbens Stapf. no agreste pernambucano. Revista Brasileira Zootecnia, v.35, n.3, p.699-705, 2006. 